\title{
Never again. Once used for cardiac catherization the Radial Artery cannot be used for CABG.
}

\author{
David Chadow ${ }^{1}$, Giovanni J. Soletti ${ }^{1}$, and Mario Gaudino ${ }^{2}$ \\ ${ }^{1}$ Weill Cornell Medicine \\ ${ }^{2}$ NewYork-Presbyterian Hospital/Weill Cornell Medical Center
}

September 28, 2021

\begin{abstract}
The use of the Radial Artery (RA) as a conduit in coronary artery bypass grafting (CABG) has been steadily increasing since the early 1990's and based on the most recent data may well become the standard of care for patients with multi-vessel coronary artery disease (CAD) requiring multiple arterial grafts. The TRA approach for cardiac catherization has also increased steadily in use by interventional cardiologists owing to its reduction in bleeding and vascular complications when compared with the femoral approach and is now considered the preferred arterial access. However, prior use of transradial access (TRA) for cardiac catherization is a contra-indication for the use of the RA for CABG because of high rates of structural damage to the vascular wall and potential for graft failure. In this issue of the Journal of Cardiac Surgery Clarke et al. examine the RA of two patients who had TRA for coronary angiography 8 and 12 years prior and note that both patients had chronic injury with dissection and obstruction of the lumen secondary to fibrosis suggesting that TRA causes long-term and irreversible damage rendering them unsuitable as conduits for CABG.
\end{abstract}

Title: Never again. Once used for cardiac catherization the Radial Artery cannot be used for CABG.

Running title: RA cath prevents use in CABG even long term.

Authors: David Chadow, $\mathrm{MD}^{1}$, Giovanni J. Soletti, $\mathrm{MD}^{1}$, Mario Gaudino, $\mathrm{MD}, \mathrm{PhD}^{1}$

${ }^{1}$ Department of Cardiothoracic Surgery, Weill Cornell Medicine, New York, NY, USA

Conflict of Interest: None

Funding : None

Disclosures : None

Article word count : 533

IRV Information: None required

Address for correspondence :

Mario Gaudino, MD, PhD

Weill Cornell Medicine, Department of Cardiothoracic Surgery,

525 E 68th St, New York, NY 10065

Phone +1 2127469440

Fax +12127468080 
Email mfg9004@med.cornell.edu

The use of the Radial Artery (RA) as a conduit in coronary artery bypass grafting (CABG) has been steadily increasing since the early 1990's and based on the most recent data may well become the standard of care for patients with multi-vessel coronary artery disease (CAD) requiring multiple arterial grafts. ${ }^{1,2}$ The use of the RA confers a morbidity and mortality benefit over the use of the saphenous vein for CABG. ${ }^{1,2}$ The most recent Guidelines on myocardial revascularization recommend the RA as the first choice for grafting the second most important coronary artery (Class I, LOE B). ${ }^{3}$

The TRA approach for cardiac catherization has also increased steadily in use by interventional cardiologists owing to its reduction in bleeding and vascular complications when compared with the femoral approach ${ }^{4}$ and is now considered the preferred arterial access according to the 2018 ESC/EACTS Guidelines on myocardial revascularization (Class $1, \mathrm{LOE} \mathrm{A})^{3}$.

However, prior use of transradial access (TRA) for cardiac catherization is a contra-indication for the use of the RA for CABG because of high rates of structural damage to the vascular wall and potential for graft failure. ${ }^{5}$ TRA leads to significantly high rates of intimal hyperplasia, impaired vasomotor function, intimal tearing, dissection, and decreased diameter, which all contribute to higher rates of graft failure. ${ }^{5}$

Although there are plenty of studies looking into the effects of TRA on the RA ${ }^{5}$, there are only two studies that specifically examined the effect of TRA on RA CABG graft function and patency when used in CABG and these studies looked only at short- and medium-term outcomes. ${ }^{6,7}$ Kamiya et al. ${ }^{6}$ found rates of stenosis free patency were more that $20 \%$ lower in those patients who had prior TRA when compared with control (77\% vs $98 \%$ ) and that intimal hyperplasia rates were $30 \%$ greater in the RA of patients who underwent TRA compared to those who did not (68\% vs $30 \%)$. Ruzieh et al. ${ }^{7}$ had similar results with patency rates that were nearly $20 \%$ lower in patients who had prior TRA when compared to patients who did not.

At the moment, there is very limited evidence on the long-term impact of TRA on the RA. Although the consensus is that the RA should not be used early following TRA, because of the lack of data questions linger if in the long term the RA will heal and be able to be used as a CABG conduit.

In this issue of the Journal of Cardiac Surgery Clarke et al. ${ }^{8}$ examine the RA of two patients who had TRA for coronary angiography 8 and 12 years prior and note that both patients had chronic injury with dissection and obstruction of the lumen secondary to fibrosis suggesting that TRA causes long-term and irreversible damage rendering them unsuitable as conduits for CABG.

More data is needed but it appears that at least some of the damage caused by TRA is permanent, and therefore the RA should not be used for CABG even late after TRA. Due to the increasing use of the RA for both percutaneous intervention and CABG this is an issue that will not go away and close coordination and teamwork is needed between clinical and interventional cardiologists and the cardiac surgery team.

1. Gaudino M, Rahouma M, Abouarab A, Leonard J, Kamel M, Di Franco A, et al. Radial artery versus saphenous vein as the second conduit for coronary artery bypass surgery: A meta-analysis. The Journal of Thoracic and Cardiovascular Surgery. 2019 May 1;157(5):1819-1825.e10.

2. Gaudino M, Benedetto U, Fremes S, Biondi-Zoccai G, Sedrakyan A, Puskas JD, et al. Radial-Artery or Saphenous-Vein Grafts in Coronary-Artery Bypass Surgery. New England Journal of Medicine. 2018 May 31;378(22):2069-77.

3. Neumann F-J, Sousa-Uva M, Ahlsson A, Alfonso F, Banning AP, Benedetto U, et al. 2018 ESC/EACTS Guidelines on myocardial revascularization. European Heart Journal. 2019 Jan 7;40(2):87-165.

4. Valgimigli M, Gagnor A, Calabró P, Frigoli E, Leonardi S, Zaro T, et al. Radial versus femoral access in patients with acute coronary syndromes undergoing invasive management: a randomised multicentre trial. Lancet. 2015 Jun 20;385(9986):2465-76. 
5. Mounsey CA, Mawhinney JA, Werner RS, Taggart DP. Does Previous Transradial Catheterization Preclude Use of the Radial Artery as a Conduit in Coronary Artery Bypass Surgery? Circulation. 2016 Aug $30 ; 134(9): 681-8$.

6. Kamiya H, Ushijima T, Kanamori T, Ikeda C, Nakagaki C, Ueyama K, et al. Use of the radial artery graft after transradial catheterization: is it suitable as a bypass conduit? Ann Thorac Surg. 2003 Nov;76(5):1505-9.

7. Ruzieh M, Moza A, Bangalore BS, Schwann T, Tinkel JL. Effect of Transradial Catheterisation on Patency Rates of Radial Arteries Used as a Conduit for Coronary Bypass. Heart, Lung and Circulation. 2017 Mar $1 ; 26(3): 296-300$.

8. Clarke N, Jagannathan G, Lawton J. Long-term consequences of trans-radial catheterization on the radial artery. Journal of Cardiac Surgery. 